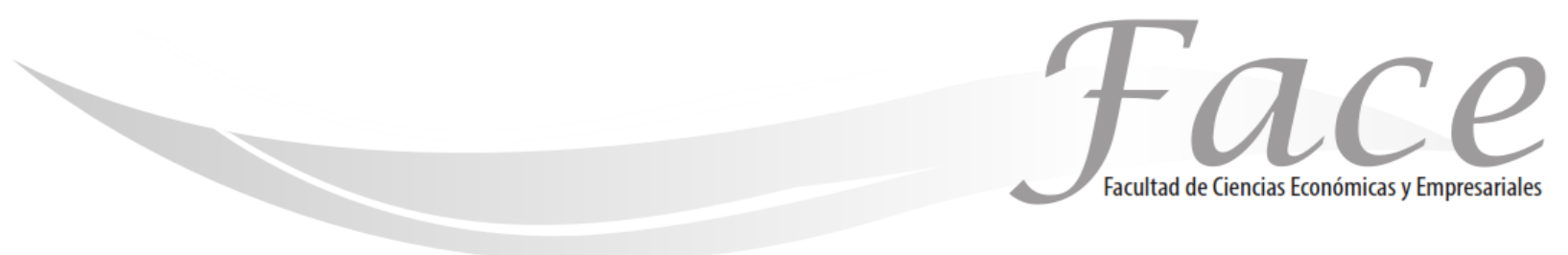

ISSN Impreso: 1794-9920

ISSN Electrónico: 2500-9338

Volumen $17-\mathrm{N}^{\circ} 2$

Año 2017

Págs. 6 - 14

\title{
LA SEGMENTACIÓN EN LA COMPRA DE PRODUCTOS SOCIALMENTE RESPONSABLES EN COLOMBIA Y SU IMPACTO EN EL MARKETING*
}

\author{
Diana María López Celis ** \\ Enlace ORCID: http://orcid.org/0000-0002-7949-0625 \\ Mónica Eugenia Peñalosa Otero*** \\ Enlace ORCID: http://orcid.org/0000-0002-2208-9224
}

Fecha de Recepción: 6 de Marzo 2017

Fecha de Aprobación: 27 de Mayo 2017

\section{Resumen:}

Esta investigación se realizó con el fin de identificar el perfil demográfico del comportamiento de los colombianos respecto a la compra de productos socialmente responsables. Es de tipo descriptivo con alcance cuantitativo, para ello se realizaron 400 encuestas a nivel nacional, teniendo en cuenta las principales ciudades capitales colombianas; Bogotá Cali, Medellín y Barranquilla, considerando los estratos socioeconómicos desde el 2 y hasta el 6, en personas mayores de 18 años, clasificadas por rangos, con una confiabilidad del $95 \%$ y un margen de error del $5 \%$. Para la recolección de la información y el trabajo de campo se contrató una empresa especializada; B\&Optimos; quienes a su vez aplicaron una encuesta estructurada auto aplicada en centros de gran afluencia. El estudio arrojó resultados interesantes, en los cuales se pudo determinar que hay una leve diferencia entre hombres y mujeres, en cuanto a la compra de productos socialmente responsables, así como una predisposición mayor en las personas ubicadas en los rangos de edad más jóvenes y respecto a la ubicación geográfica. Medellín presenta los índices más altos respecto a otras ciudades en cuanto a este tipo de compra. Por otra parte, se identificó que los productos en donde las personas tienen en cuenta las características de los productos socialmente responsables con un mayor porcentaje o participación son alimentos, ropa y tecnología.

Palabras Clave: Consumo socialmente responsable, segmentación demográfica, productos, servicios.

\footnotetext{
* Artículo resultado de la investigación titulada Medición y caracterización del Consumo Socialmente Responsable en Colombia, avalada por la convocatoria No. 12 de la Dirección de Investigaciones de Utadeo.

**Profesora Asociada de la Facultad de Ciencias Económicas y Administrativas, Universidad Jorge Tadeo Lozano, Bogotá, Colombia. Magister en Psicología del Consumidor, especialista en Psicología del Consumidor. Profesional en Psicología. Investigadora Jr. de Colciencias. Se ha venido desempeñando en los últimos años como Docente - Investigadora en la Universidad Jorge Tadeo Lozano, la Universidad Central y la Universidad Javeriana- Colombia. Correo electrónico: diana.lopez@utadeo.edu.co

${ }^{* * *}$ Profesora Asociada de la Facultad de Ciencias Económicas y Administrativas, Universidad Jorge Tadeo Lozano, Bogotá, Colombia. Magister en Mercadeo Agroindustrial. Profesional en Relaciones Internacionales. Investigadora Jr. de Colciencias. Se ha venido desempeñando en los últimos años como Docente Investigadora en la Universidad Jorge Tadeo Lozano, la Universidad de La Salle y la Universidad de Pamplona - Colombia. Correo electrónico: monica.penalosa@utadeo.edu.co
} 


\title{
SEGMENTATION IN THE PURCHASE OF SOCIALLY RESPONSIBLE PRODUCTS IN COLOMBIA AND ITS IMPACT ON MARKETING
}

\begin{abstract}
This research was conducted in order to identify the demographic profile of the behavior of Colombians regarding the purchase of socially responsible products. It is of a descriptive type with a quantitative scope, for which 400 surveys were carried out nationwide, taking into account the main Colombian capital cities; Bogotá Cali, Medellín and Barranquilla, considering socioeconomic strata from 2 to 6 , in people over 18 years, classified by ranges, with a reliability of $95 \%$ and a margin of error of $5 \%$. For the collection of information and field work a specialized company was hired; B \& Optimas; who in turn applied a structured self-administered survey in high-traffic centers. The study yielded interesting results, in which it was possible to determine that there is a slight difference between men and women, in terms of the purchase of socially responsible products, as well as a greater predisposition in the people located in the youngest age ranges and to the geographical location. Medellín has the highest rates compared to other cities in terms of this type of purchase. On the other hand, it was identified that products where people take into account the characteristics of socially responsible products with a higher percentage or participation are food, clothing and technology.
\end{abstract}

Keywords: Socially responsible consumption, demographic segmentation, products, services.

\section{SEGMENTAÇÃO NA COMPRA DE PRODUTOS SOCIALMENTE RESPONSÁVEIS NA COLÔMBIA E SEU IMPACTO NO MARKETING}

\section{RESUMO}

Esta pesquisa foi realizada com o objetivo de identificar o perfil demográfico do comportamento dos colombianos em relação à compra de produtos socialmente responsáveis. Trata-se de um tipo descritivo de alcance quantitativo, para o qual foram realizadas 400 pesquisas em todo o país, levando em conta as principais capitais colombianas; Bogotá Cali, Medellín e Barranquilla, considerando os estratos socioeconômicos de 2 a 6 , em maiores de 18 anos, classificados por faixas, com confiabilidade de $95 \%$ e margem de erro de 5\%. Para a coleta de informações e trabalhos de campo, foi contratada uma empresa especializada; B \& Optimas; que por sua vez aplicou uma pesquisa auto-administrada estruturada em centros de alto tráfego. $O$ estudo produziu resultados interessantes, que poderia ser determinado que há uma ligeira diferença entre homens e mulheres em relação à compra de produtos socialmente responsáveis e uma maior vontade em pessoas localizadas nas fileiras da idade mais jovem e respeito para a localização geográfica. Medellín tem as taxas mais altas em comparação com outras cidades em termos desse tipo de compra. Por outro lado, identificou-se que os produtos em que as pessoas levam em consideração as características de produtos socialmente responsáveis e com maior percentual ou participação são alimentos, roupas e tecnologia.

Palavras-chave: Consumo socialmente responsável, segmentação demográfica, produtos e serviços. 


\section{INTRODUCCIÓN:}

El Consumo Socialmente Responsable (CnSR), puede ser entendido como un cambio de paradigma que se ha venido presentando alrededor del mundo, se trata de una modificación en el pensamiento de los consumidores que los hace ser más conscientes acerca de lo que consumen y la manera en la que lo hacen. El CnSR es ampliamente conocido a nivel mundial, especialmente en Europa, y en Estados Unidos, en donde se han podido identificar comportamientos, perfiles y rasgos característicos de los consumidores que siguen este modo de vida; tanto a nivel demográfico como a nivel psicográfico, caracterización que cobra relevancia no solamente desde el punto de vista de conocimiento sino de las implicaciones que puede tener en el marketing.

Tener un conocimiento generalizado respecto a la caracterización demográfica del consumidor Colombiano que compra productos socialmente responsable puede resultar de gran ayuda en el momento de la planeación estratégica para el posicionamiento de marca, este estudio es una aproximación al conocimiento general del consumidor frente a este aspecto puesto que un análisis completo debe involucrar un conocimiento de este tipo de consumidor a nivel psicográfico y un abanico más amplio de variables demográficas. Este planteamiento trae como consecuencia un desarrollo para empresas socialmente responsables, como lo justifica Hernández, y otros (2014), el desarrollo institucional es propio del proceso evolutivo hacia el surgimiento de un mercado formal.

\section{MARCO TEÓRICO:}

\section{Caracterización del Consumo Socialmente Responsable en el mundo}

A nivel mundial se pueden encontrar distintos estudios acerca del tema; según Dueñas et al., (2014 p. 293), se encuentra un estudio desarrollado en Malasia (2006), llamado Escala del Consumismo Ambiental y Comportamiento de Compra, en donde subdivide los elementos relacionados con el consumo responsable entre micro factores y macro factores, siendo los primeros aquellos relacionados con el comercio justo y los segundos los aspectos medioambientales más relevantes.
En un estudio realizado por Anderson y Cunningham, citado por Frederick et al., (1975, p.189) se encontró que entre las características de los consumidores socialmente responsables se encontró que eran jovenes adultos, con altos cargos porfesionales y nivel socioeconómico, también eran mas cosmopolita, menos dogmáticos y menos alienados. Esta caracterización se contradice con los resultados arrojados por la escala de (ERS). Igualmente, en Hong Kong (2008) se desarrolló una escala que medía Influencia social, actitud ambiental, preocupación ambiental, seriedad percibida sobre los problemas ambientales, responsabilidad ambiental percibida, efectividad percibida sobre el efecto que tiene un comportamiento ambientalista. (Dueñas et al., 2014 p. 294). Así mismo, en países como la India (2009), Corea del Sur (2010) y China (2011), desarrollaron escalas que a nivel general medían intención de compra, protección ambiental percepción sobre la responsabilidad social empresarial, consumo moderado entre otras. (Dueñas et al., 2014 p. 295).

Según Dueñas et al., (2014 p. 293), en Francia (2006), se desarrolló la escala de Consumo Socialmente Responsable. En relación al consumidor español según Llopis(2009, p.160), se muestra una creciente sensibilidad en relación con la decisión de compra, en donde no solo se tienen en cuenta factores como precio, calidad y distribución, sino que además cómo los productos han sido fabricados, y quién se beneficia con su compra. Burns (1995), citado por Llopis (2009, p.160). Las variables que se utilizaron para medir el consumo responsable en cuanto al comercio justo, se dividen en tres: la primera se refiere a Sentimiento de Eficacia (eficacia presente y futura de las ONG para la solución de problemas en los países en vías de desarrollo), la segunda habla de Donaciones Economicas o Compasión (apoyo a campañas comunitarias) y finalmente la Orientación Cognitiva Global (interés por la procedencia geográfica de los productos adquiridos).

Los resultados de este estudio mostraron que la tendencia en las actitudes de los consumidores españoles tienen una Orientación Cognitiva Global que está condicionada por elementos normativos, esto quiere decir que "su surgimiento haría posible que los individuos tomen conciencia del origen de los problemas que les afectan, ya sean estos de índole socioeconómica, ecológica o política". Rosenau (1990, p.335), citado por Llopis (2009, p.161).

De acuerdo con Valor \& Calvo (2009, pp.34-35) define a los consumidores españoles como individuos que no conocen muy bien acerca del consumo socialmente responsable pues no hay información disponible en los puntos de venta del producto, también resaltan que a pesar de esta situación 
se puede denotar que hay un interés muy fuerte hacia la adopción de prácticas de consumo responsable lo cual les impulsa a buscar más información. Como lo define Carrero et al., (2015, p.4) "El consumo responsable no es un fenómeno homogéneo en cuanto a la motivación fundamental, ni en cuanto a las variables relativas a la psicología del individuo, ni en el tipo y forma de procesamiento de la información, ni en la conducta". En cuanto al consumidor Latinoamericano, García (1995), lo define como un consumidor que está siendo evaluado a través de sus características pscicográficas (personalidad, estilo de vida, valores actitudes y intereses) y sus características demográficas (edad, género, nivel ,educativo, ingresos,status socio-economico y cultura).

Respecto a la medición del consumidor colombiano se encuentra un estudio que evalúa fenómenos de Conciencia social, Servicio al cliente, Consumo Responsable 0 Sostenible. Esta investigación cuenta con una metodología cualitativa de tipo descriptivo exploratorio, compuesta por estrevistas semi estructuradas construidas apartir de la matriz de necesidades."Dentro del instrumento se incluyeron preguntas basadas en varias de las categorías planteadas por Max Neef; estas son subsistencia, protección, afecto, entendimiento y ocio" (García \& Betancourt, 2014).

A su vez, se evidenció que existen algunas herramientas para identificar a un consumidor responsable en Colombia, entre ellas se encuentran la compra de productos y sevicios sostenibles, manejo de residuos y deshechos, promoción de la responsabilidad social y el activismo Yepes (2012); en ella, hacen un desarrollo de las actividades que se encuentran dentro de estas herramientas. A continuación se hace una breve mención de cada una.

En cuanto a la compra de producto y servicios sostenibles encuentran actividades como la adquisición de bienes sanos y seguros, que generen energía limpia, que pertenezcan al comercio justo, y que tengan prácticas inclyentes, todo lo relacionado a actividades que involucren la adquisición y el consumo en general de productos se encuentra en esta herramienta para ser un consumidor responsable. Para el manejo de residuos se encuentra compuesto por las tres r's del reciclaje en donde se pueden encuentrar: reciclar, reusar y reducir. Igualmente se encuentran el recuperar productos (reformarlos) y rechazar; esta última actividad se refiere principalmente a abstenerce de adquirir productos que hagan daño al medio ambiente. Estas actividades hacen referencia al deshecho de los productos.
Con respecto a la segmentación del consumidor socialmente responsable y de la mano con los estudios ya mencionados, se encuentra que se debe tener en cuenta un mix entre variables demográfica y psicográficas, debido a que no existe evidencia que apunte a concretar cuál es más importante. Al referenciar el estudio realizado en China por (Zhilong Tian, Rui Wang, Wen Yang, 2011) sobre la segmentación demográfica del consumidor socialmente responsable, se demuestra que la relación entre las variables demográficas y CSR no son lineales de acuerdo a los resultados de las regresiones realizadas; por otra parte (Pérez y Rodríguez 2015) exponen la forma en que los consumidores construyen imágenes de responsabilidad social, teniendo en cuenta las variables demográficas, como moderadoras dentro del estudio. En el caso de género, algunos estudios concluyen que los hombres tienen un mayor conocimiento respecto al tema (Mostafa 2007); así mismo, el estudio de (Roberts 1995) identifica un mayor conocimiento por parte de las mujeres, lo cual ha sido expuesto por (Carrete, Gonzáles \& colegas 2013) en el Think Green en México, sin desconocer que la segmentación demográfica es importante para poder acceder al mercado potencial. (Pascual, Peñalosa \& López 2015).

La segmentación demográfica es entendida como la variable que "divide a los consumidores de acuerdo con la edad, el genero, el origen étnico, el ingreso y riqueza, el estado civil, la ocupación, el tipo y tamaño de familia, y la ubicación geográfica" (Shiffman 2015p 29). Para la empresa al inicio del ciclo de vida de la empresa la segmenación es un tema que en algunos instacias no presenta alguna formalidad 0 sistematización, caso contrario lo plantea Arias \& Perez (2015), El emprendedor en las fases previas solo tiene hipótesis del mercado, de la industria, de sus capacidades, por lo cual es fundamental el que pueda probar cada supuesto y modicarlo para el cliente.

\section{METODOLOGÍA:}

El alcance de la investigación es cuantitativo con un enfoque descriptivo, basado en la aplicación de encuestas autoadministradas a nivel nacional para la recogida de la información. Variables demográficas, estrato (nivel socioeconómico del 3 al 6), edad (entre 18 y 60años), ciudad (Bogotá, Cali, Medellín y Barranquilla), género (femenino y masculino) y la distribución a través de la compra de productos socialmente responsables. 
Se realizó un muestreo no probabilístico en centros de gran afluencia, para la selección de los participantes que formaron parte del estudio se utilizó un muestreo estratificado por género y estrato socioeconómico, el trabajo de campo fue realizado por B\&Optimos; en el segundo semestre de 2015, las entrevistas tuvieron un tiempo aproximado de 20 minutos.

La investigación se realizó con una muestra de 400 sujetos con una confiabilidad del $95 \%$ y $5 \%$ de margen de error teniendo en cuenta el Universo total de la población Colombiana 48.010.049 (fuente Dane), distribuidas proporcionalmente para cada ciudad; 100 en Cali, 100 en Bogotá, 100 en Barranquilla y 100 en Medellín. Este muestra garantizó un factor de muestreo situado por debajo del $8-9 \%$, lo que sitúa la representatividad de la muestra en los términos aceptados y validados en la literatura previa (Román \& Cuestas, 2008; Román, 2010).

\section{RESULTADOS Y DISCUSIÓN:}

De acuerdo con el trabajo de campo realizado, a continuación se presenta los resultados más representativos.

Tabla 1. Compra de productos Socialmente Responsables

¿Ha comprado alguna vez algún producto socialmente responsable?

\begin{tabular}{llr|r|r|r} 
& & Frecuencia & Porcentaje & \multicolumn{1}{c}{$\begin{array}{c}\text { Porcentaje } \\
\text { válido }\end{array}$} & $\begin{array}{c}\text { Porcentaje } \\
\text { acumulado }\end{array}$ \\
\hline Válido & Si & 233 & 58,2 & 58,2 & 58,2 \\
\cline { 2 - 6 } & No & 167 & 41,8 & 41,8 & 100,0 \\
\cline { 2 - 6 } & Total & 400 & 100,0 & 100,0 & \\
\hline
\end{tabular}

Más del $50 \%$ de la población manifiesta comprar productos socialmente responsables, distribuida demográficamente de la siguiente manera. Medellín es la ciudad con una mayor disposición a comprar productos socialmente responsables; mientras que Bogotá presenta la menos disposición.

Tabla 2. Disposición de Compra por Género

\begin{tabular}{|c|c|c|c|}
\hline & Hombre & Mujer & Total \\
\hline Frecuencia & 120 & 113 & 233 \\
\hline Porcentaje & $51.5 \%$ & $48.5 \%$ & $100.0 \%$ \\
\hline
\end{tabular}

Fuente: elaboración propia
Con una diferencia de 2 puntos porcentuales, los hombres presentan una mayor disposición a la compra de productos socialmente responsables.

\section{Tabla 3. Compra de Productos por edades}

\begin{tabular}{|l|c|c|c|c|c|c|}
\hline & Entre 18 y 25 años & Entre 26 y 35 años & Entre 36 y 45 años & Entre 46 y 55 años & Entre 56 y 60 años & Total \\
\hline Frecuencia & 104 & 57 & 32 & 29 & 11 & 233 \\
\hline Porcentaje & $44.6 \%$ & $24.5 \%$ & $13.7 \%$ & $12.4 \%$ & $4.7 \%$ & $100.0 \%$ \\
\hline
\end{tabular}

Fuente: elaboración propia

La población joven principalmente muestran el porcentaje más alto en cuanto a la compra de productos socialmente responsables.

\section{Tabla 4. Compra por nivel socioeconómico}

\begin{tabular}{|c|c|c|c|c|c|}
\hline & Estrato 3 & Estrato 4 & Estrato 5 & Estrato 6 & Total \\
\hline Frecuencia & 135 & 50 & 31 & 17 & 233 \\
\hline Porcentaje & $57.9 \%$ & $21.5 \%$ & $13.3 \%$ & $7.3 \%$ & $100.0 \%$ \\
\hline
\end{tabular}

Fuente: elaboración propia

En cuanto a nivel socioeconómico, la clase media y media alta presentan el mayor porcentaje en cuanto a compra de productos socialmente responsables.

\section{Gráfico 5. Tipos de Productos Socialmente Responsables adquiridos}

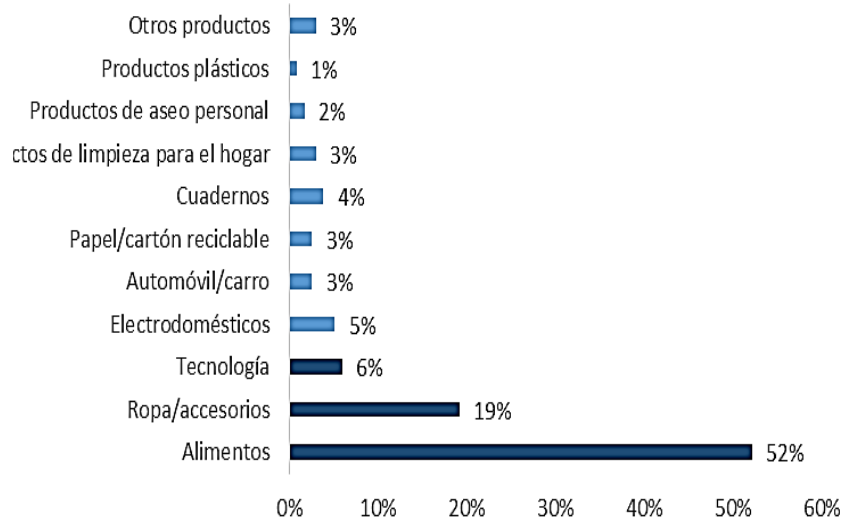

Fuente: elaboración propia

Los productos en donde se identifica una mayor tendencia a contemplar las características de los productos socialmente responsables, son alimentos y vestuario seguidos de tecnología. 


\section{CONCLUSIONES:}

De manera general con respecto a la compra de bienes en Colombia, encontramos que en la totalidad de la población existe un porcentaje superior (equivalente al $50 \%$ ) en la compra de productos socialmente responsables, lo cual es un indicador positivo, teniendo en estudios previos en donde se argumenta, que los hombres tienen una mayor disposición a la compra de este tipo de productos en línea

con el estudio de (Mostafa 2007), el cual concluye precisamente que los hombres tienen un mayor conocimiento respecto al tema.

Se evidenció, que los jóvenes específicamente con un rango de edades comprendido entre 18 y 25 años, es la población de mayor predisposición a la compra de este tipo de productos; lo cula se alínea con el estudio de Anderson y Cunningham, citado por Frederick et al., (1975, p.189), en el cual se encontró también que entre las características demográfica de los consumidores socialmente responsables, es el grupo más susceptible a las compras con esta característica específica, lo cual representa una gran oportunidad para el mercadeo porque se puede cultivar en este target dicha tendencia y ser aprovechada como ventaja competitiva.

Al realizar el análisis teniendo en cuenta la segmentación demográfica, específicamente la ubicación geográfica, podemos encontrar identificadas claramente las zonas en las cuales se encuentra una mayor probabilidad de compra hacia productos socialmente responsable, destacándose principalmente Medellín y Cali con promedios superiores y Bogotá con uno de los porcentajes más bajos, al igual que Barranquilla, lo cual resulta un tema de interés para futuras investigaciones, siendo importante profundizar en cuanto a las políticas gubernamentales, las estrategias de comunicación o los mismos programas de responsabilidad social de las empresas; porque se demuestra que no están impactando en todas las zonas geográficas de la misma manera y claramente se determina que los planes de marketing deben ser estratégicamente diferenciados de acuerdo a esta variable. Tambien mencionado en el sector industrial por Martínez, A. G. C. (2016).
Por último, es clara la responsabilidad y la oportunidad que tienen las empresas Colombianas en el sector de los alimentos, el vestuario y la tecnología, teniendo en cuenta que son los productos en donde los consumidores prestan una mayor atención a las características generales respecto a la responsabilidad social.

Es claro de acuerdo a la revisión bibliográfica, que la predisposición a la compra de productos socialmente responsables es un análisis en donde tanto las variables psicográficas como las demográficas se complementan, pero es indiscutible que la segmentación demográfica ayuda a focalizar y optimizar los recursos en un plan de mercadeo, donde se pretende potencializar la predisposición de los consumidores a la compra de productos socialmente responsables. "No hay duda de que el consumo responsable ha de ser co-creado por las empresas $y$ losdirectivos" Caruana \& Crane (2008), citado por Smith \& Williams, (2011, p.76). 


\section{REFERENCIAS:}

Alto Nivel. (s.f.). Recuperado el Junio de 2015, de ALTO NIVEL: http://www.altonivel.com.mx/36230-7grandes-tendencias-de-marketing-para-al.html

Arias, M., \& Perez, R. (2015). Emprendimiento: como comenzar una empresa con éxito. Face: Revista de la Facultad de Ciencias Económicas y Empresariales, 14, 175-183.

Barragán, A. (2015). Ventajas de la Responsabilidad Social Empresarial. PYMERANG.

Brundtland, G. (1987). Nuestro Futuro en Común. Naciones Unidas.

Capriotti, P. (2009). Branding Corporativo fundamentos para la gestión estratégica de la identidad corporativa. Chile: Colección de Libros de la Empresa.

Carrero, I., Bilbao, P., Díaz, E., Labajo, V., Merino, A., \& Valor, C. (2015). El despertar al consumo responsable: análisis de un proceso de crecimiento. Cantabria: Universidad Pontifica.

Caruana, R., \& Crane, A. (2008). Constructing Consumer Responsibility: Exploring the Role of Corporate Communications. Organization Studies, 14961519.

Centro Andino de Integración. (s.f.). Centro Andino de Integración. Obtenido de PROTOCOLOS Y CONVENIOS SOBRE EL MEDIO AMBIENTE: http://centroandinodeintegracion.org/protocolosmedio-ambiente/

Comercializadora de soluciones informáticas. (s.f.). CSI S.A. Recuperado el Junio de 2015, de CSI S.A.

Consumo Sostenible. (s.f.). Consumo Sostenible- Tu compra es tu decisión. Recuperado el 5 de Junio de 2015, de Consumo Sostenible- Tu compra es tu decisión: http://www.consumosostenible.co/perfil-comoconsumidor-3?view=quiz
Consumoresponsable.org. (2011). Criterios para un consumo responsable. Obtenido de http://www.consumoresponsable.org/criterios/inde $\mathrm{x}$

DANE. (2005). Cuadro censo 2005. DANE.

Dinero. (1 de Enero de 2014). Los mitos y verdades del consumidor colombiano. Recuperado el 8 de Junio de 2015, de Dinero: http://www.dinero.com/pais/articulo/los-mitosverdades-del-consumidor-colombiano-estudiokantar-worldpanel/201574

Dueñas, S., Perdomo, J., \& Villa, L. (2014). El concepto de consumo socialmente responsable y su medición. Una revisión de la literatura. Estudios Gerenciales, 287-300.

España, R. (s.f.). FENALCO. Recuperado el 8 de Junio de 2015, de FENALCO: http://www.fenalco.com.co/contenido/2852/Los\%2 Ocambiantes $\% 20 \mathrm{~h} \% \mathrm{C} 3 \% \mathrm{~A} 1$ bitos $\% 20$ del $\% 20$ cons umidor $\% 20$ colombiano

Frederick, E., \& Webster, J. (1975). Determining the Characteristics of the Socially Conscious Consumer. Journal Of Consumer Research, 188196.

Fundación para la cooperación APY - Solidaridad en Acción. (2012). El consumo responsable como generador de cambio social. Andalucía: Fundación para la cooperación APY - Solidaridad en Acción.

García, L., \& Betancourt, D. (2014). La Racionalidad del Cconsumidor vista desde la conciencia social. Bogotá D.C: Pontificia Universidad Javeriana. 
García, N. (1995). El consumo sirve para pensar. Consumidores y ciudadanos. Conflictos Multiculturales de la globalización., 41-55.

Gómez, I. (s.f.). Los estratos socioeconómicos en Colombia. Obtenido de http://igomeze.blogspot.com/2010/06/los-estratossocioeconomicos-de.html

Hernández, I. D., Álvarez, R., Blanco, C., \& Carvajal, A. (2014). El ascenso de la "mano invisible": análisis para el surgimiento de un mercado formal de financiación para empresas de base tecnológica (ebt) en Colombia. FACE: Revista de la Facultad de Ciencias Económicas y Empresariales, 13(1), 5-32.

Hoyer, W., \& Maclnnis, D. (2010). Comportamiento del Consumidor- Quinta edición. México: Cenegage Learning Editores S.A.

IESE Business School. (2009). La evolución del concepto de los Stakeholders en los escritos de Ed Freeman. Newsletter $\mathrm{N}^{\circ}$ 5- Otro punto de vista, 1-4.

Leone, G. (s.f.). Leyes de la Gestalt. Obtenido de http://www.guillermoleone.com.ar/LEYES\%20DE \%20LA\%20GESTALT.pdf

Llopis, R. (2009). Consumo Responsable y Globalización Reflexiba: Un Estudio Referido Al Comerio Justo En España. Revista Española del Tercer Sector, 145-165.

Martínez, A. G. C. (2016). Acercamiento al proceso de toma de decisiones en las PYMES del sector industrial de la ciudad de Duitama, Boyacá, Colombia. FACE: Revista de la Facultad de Ciencias Económicas y Empresariales, 16(1), 4860.

Mateus, C. (2007). Estándares de buenas prácticas para la toma de muestras. Instituto Nacional de Estadística, 1-14.

Miniard, P., Blackwell, R., \& Engel, J. (2002). Comportamiento del Consumidor. Cengage Learning Editores S.A.

Nielsen, L., \& McGregor, S. (2013). Consumer morality and moral norms. International Journal of Consumer Studies, 473-480.
Oviedo, \& Paz. (7 de Octubre de 2010). El blog de Oviedo \& Paz C.A. Recuperado el 7 de Junio de 2015, de El blog de Oviedo \& Paz C.A: https://oviedoypaz.wordpress.com/2010/10/07/\% C2\%BFque-es-consumo-socialmenteresponsable/

Oxford Dictionaries . (s.f.). Moral. Obtenido de http://www.oxforddictionaries.com/es/definicion/es panol/moral

Pascual del Riquelme Martínez, M., \& Peñalosa Otero, M., \& López Célis, D. (2015). El consumo socialmente responsable en el mercado colombiano. Cuadernos Latinoamericanos de Administración, XI (21), 61-70.

Schiffman, L., \& Kanuk, L. (2005). Comportamiento del consumidor -Octava edición. México: Pearson.

Smith, N., \& Williams, E. (2011). Responsible consumers and stakeholder marketing: building a virtuous circle of social responsibility. Universia Business Review, 68-78.

Sostenibilidad.com. (s.f.). ¿Sabes cuándo nace la sostenibilidad? Obtenido de Sostenibilidad para todos: http://www.sostenibilidad.com/sabescuando-nace-la-sostenibilidad/

Uribe, C. (2008). Estratificaión Social en Bogotá: de la política pública a la dinámica de la segregación social. Universitas humanística no.65, 139-171.

Valor, C., \& Calvo, G. (2009). Compra Responsable en España. Comunicación de Atributos Sociales y Ecológicos. Boletín Económico de ICE N²971, 33-50.

Yepes, G. (29 de Diciembre de 2012). Consumo Responsable Fundamentos y práctica. Obtenido de supersociedades.gov.co: http://www.supersociedades.gov.co/inspeccionvigilancia-y-control/gobierno-corporativo-yrse/documentos/Documentos\%20RSE/Consumo_ Responsable_Nov_2012\%20(7).pdf

García Canclini Néstor 1995, Consumidores y ciudadanos, conflictos multiculturales de la globalización, Editorial Grijalbo S.A pag 41-55 
ISSN: 1794-9920 Enero - Abril de 2017

Volumen 17 Número 2, Año 2017 Págs. 6 - 14

Leon G Shiffman. Joseph Wisenblit 2015 Comportamiento del consumidor décimo primera edición, editorial Pearson Página 29. 